\title{
Effect of preoperative administration of systemic alpha-2 agonists on postoperative pain: a systematic review and meta-analysis
}

\author{
Ji Youn Ju, Kye-Min Kim, and Sangseok Lee
}

Received September 2, 2019

Revised October 20, 2019

Accepted October 25, 2019

Department of Anesthesiology and Pain Medicine, Sanggye Paik Hospital, Inje University

College of Medicine, Seoul, Korea

\author{
Corresponding author \\ Sangseok Lee, M.D. \\ Department of Anesthesiology and \\ Pain Medicine, Sanggye Paik Hospital, \\ Inje University College of Medicine, \\ 1342 Dongil-ro, Nowon-gu, Seoul \\ 01757, Korea \\ Tel: 82-2-950-1171 \\ Fax: 82-2-950-1323 \\ E-mail: s2248@paik.ac.kr
}

\begin{abstract}
Background: Alpha-2 agonists have sedative, analgesic, and opioid-sparing effects. Moreover, intraoperative or postoperative systemic administration of alpha-2 adrenergic agonists is known to reduce postoperative pain and opioid consumption. This meta-analysis investigated whether preoperative administration of alpha-2 agonists can affect postoperative pain and opioid consumption.
\end{abstract}

Methods: We searched the MEDLINE, EMBASE, Cochrane Library (CENTRAL), KoreaMed, and KMbase databases through March 2019 to identify relevant randomized controlled trials (RCTs) on the effect of preoperative systemic administration of alpha-2 agonists on postoperative pain and opioid consumption. We conducted a meta-analysis according to the Cochrane Collaboration guidelines.

Results: Eleven RCTs involving 748 participants were included in this meta-analysis. Preoperative administration of systemic alpha- 2 agonists significantly reduced cumulative opioid consumption up to $6 \mathrm{~h}$ (SMD, $-0.52 ; 95 \%$ confidence interval [- 0.90 to -0.14$]$ ) and $24 \mathrm{~h}$ (SMD, -0.68 [-1.27 to -0.09$]$ ) after surgery. Moreover, preoperative administration of alpha-2 agonists significantly reduced postoperative pain intensity at $6 \mathrm{~h}$ (SMD, -0.50 [-0.78 to -0.21$])$ and $24 \mathrm{~h}$ (SMD, -0.44 [-0.86 to -0.03$])$.

Conclusions: In this meta-analysis, high degree of heterogeneity limits the preoperative administration of alpha- 2 agonists in reducing postoperative opioid consumption and pain intensity. Future powered large RCTs are required to increase the certainty of evidence on the effect in reducing postoperative opioid consumption and pain intensity.

Keywords: Adrenergic alpha-2 receptor agonists; Anesthesia; Clonidine; Dexmedetomidine; Meta-analysis; Analgesics, opioid; Postoperative pain; Systematic review.

\section{INTRODUCTION}

Alpha-2 adrenergic agonists, which are widely used for various purposes. As an anesthetic premedication, alpha-2 agonists mainly have anxiolytic effects and have the effect of keeping hemodynamic index stable and reducing the amount of anesthetics during surgery. Also, intraoperative alpha-2 agonists is often administered to perform sedation during regional anesthesia. Because of their analgesic properties, intraoperative or postoperative use of alpha-2 agonists may be good adjuvants for use in postoperative analgesia and may reduce consumption of analgesics $[1,2]$. Recently, multimodal analgesia with reduced doses of opioids has been emphasized since increased doses of opioids lead to adverse events, such as excessive sedation, hypersensitivity, postoperative nausea, vomiting, and respiratory

This is an Open Access article distributed under the terms of the Creative Commons Attribution Non-Commercial License (http://creativecommons.org/licenses/by-nc/4.0) which permits unrestricted non-commercial use, distribution, and reproduction in any medium, provided the original work is properly cited.

Copyright (c) the Korean Society of Anesthesiologists, 2020 
depression $[1,3]$.

A previous meta-analysis demonstrated that intraoperative or postoperative systemic administration of alpha- 2 adrenergic agonists reduced postoperative pain and opioid consumption [4]. That meta-analysis, which included 30 studies, provided evidence of postoperative morphine-sparing at $24 \mathrm{~h}$ and showed that the weighted mean difference was $4.1 \mathrm{mg}$ (95\% confidence interval [CI], 6.0 to 2.2 ) with clonidine and $14.5 \mathrm{mg}$ (22.1 to 6.8) with dexmedetomidine. This meta-analysis also provided evidence of a decrease in pain intensity at $24 \mathrm{~h}$ and showed that the weighted mean difference on a 10-cm visual analog scale (VAS) was $0.7 \mathrm{~cm}$ (1.2 to 0.1 ) with clonidine and $0.6 \mathrm{~cm}$ (0.9 to 0.2 ) with dexmedetomidine.

Nevertheless, controversy exists regarding whether preoperative use of alpha-2 agonists as sedatives has an effect on reducing postoperative pain. Shariffuddin et al. [5] concluded that a single dose of dexmedetomidine was a useful adjuvant in reducing postoperative pain, and Sung et al. [6] demonstrated that oral clonidine premedication reduced the requirement of postoperative analgesia in patients undergoing laparoscopic cholecystectomy. However, Lee et al. [7] found that pre-anesthetic administration of a single loading dose of dexmedetomidine given $10 \mathrm{~min}$ before induction did not reduce patient-controlled anesthesia consumption of postoperative fentanyl or the pain score.

The aim of this study was to evaluate the effect of alpha-2 agonists administered before general anesthesia and surgery on postoperative pain and opioid consumption for postoperative analgesia.

\section{MATERIALS AND METHODS}

\section{Study design}

This systematic review and meta-analysis was performed according to recent methodological guidelines [8]. The protocol was registered on PROSPERO (https://www.crd. york.ac.uk/PROSPERO, no. CRD42016051454). The study was approved by the Institutional Review Board of the Inje University Sanggye Paik Hospital (no. 2017-08-013).

\section{Information sources and search strategy}

Three researchers (JJ, KMK, and SL) systematically searched electronic databases such as the PubMed/MEDLINE, EMBASE, and Cochrane Library (CENTRAL) without restriction on the year of publication. Additionally, we manually searched the reference lists of included studies to identify other relevant trials in the Korean databases (KoreaMed [https://koreamed.org] and KMbase [http://kmbase.medric.or.kr]). The search terms included variants of alpha-2 adrenergic receptor stimulating agents and postoperative pain, as well as Medical Subject Heading (MeSH) or EMBASE Subject Heading (EMTREE) terms. A primary investigation was performed to confirm the search keywords and strategy (Appendix 1). The language of the articles was limited to Korean and English. The last search was conducted on March 26, 2019.

\section{Study selection and eligibility criteria}

The draft search of the electronic databases for identifying potentially relevant research was completed by two authors (JJ and KMK). Thereafter, they independently selected or excluded the studies. Primary selection was based on an article's title and abstract. Secondary selection was performed after full-text review of an article. Studies for final assessment were selected in consensus by the two investigators. If necessary, a third investigator (SL) participated in study selection, and the final decision was made on the basis of a majority.

Studies were included in the meta-analysis if they satisfied the following criteria: (1) patients underwent non-cardiac surgery or neurosurgery under general anesthesia; (2) intravenous or oral administration of an alpha-2 agonist was used prior to surgery as an intervention; (3) results of the control group were reported; and (4) postoperative pain scores or opioid consumption was reported as a primary outcome.

Studies were excluded for the following reasons: (1) intranasal, rectal, local, or topical administration of an alpha-2 agonist; (2) did not report appropriate outcomes or outcome measurements as mentioned; (3) patients underwent epidural anesthesia combined with general anesthesia; (4) non-randomized controlled trials (RCTs) or studies without a control group; (5) non-human studies; and (6) articles not in Korean or English.

\section{Risk of bias in individual studies}

Two authors (JJ and KMK) reviewed the articles independently to assess the risk of bias by using the "risk of bias (ROB)" tool provided in the Review Manager software ver- 
sion 5.3 (The Cochrane Collaboration, UK) based on Cochrane's assessment of risk of bias. If necessary, a third reviewer (SL) was included to sort disagreements. The following seven domains to assess the risk of bias were used in each trial: random sequence generation, allocation concealment, blinding of participants and personnel, blinding of outcome assessment, incomplete outcome data, selective reporting, and other bias. We specified the seventh domain "other bias" for assessing the adequate estimation of sample size calculation. The methodology for each trial was graded as "high," "low," or "unclear" to reflect a high risk of bias, low risk of bias, or uncertainty of bias, respectively.

The agreement between the two independent reviewers for the level of risk of bias regarding the seven domains was assessed using Cohen's kappa [9].

\section{Data collection process and extracted items}

Three authors (JJ, KMK, and HK) extracted information from the original articles, and another author (SL) independently confirmed all the extracted data. The following data were included in the data extraction form: patient characteristics, age, sex, intervention dose and method, pain-measurement tool and pain score, and postoperative analgesic method and usage. If the data were insufficient or unclear, the data were extracted from the text, tables, or graphs.

Regarding preoperative administration of alpha-2 agonists, only intravenous (IV) or oral administration method was included, but the mode of administration (bolus or continuous infusion) and dose were not restricted. A study using continuous infusion of intervention drugs during surgery was excluded.

Before we selectively extracted data from various studies, the pain scores measured at postoperative $6 \mathrm{~h}$ or at the nearest time point within postoperative $6 \mathrm{~h}$ were defined as "early period pain scores." The scores measured at postoperative $24 \mathrm{~h}$ were defined as "late period pain scores." In case of lack of data at postoperative $24 \mathrm{~h}$, the latest pain scores between postoperative 6 and $24 \mathrm{~h}$ were considered as "late period pain scores." Cumulative opioid consumption up to $6 \mathrm{~h}$ after surgery were defined as "early period opioid consumption." If the data for cumulative opioid consumption were not available at postoperative $6 \mathrm{~h}$, superseded data were collected in the same way with those of pain scores. "Late period opioid consumption" was defined as the cumulative opioid consumption up to $24 \mathrm{~h}$ after surgery.

The VAS scores were extracted for analyzing postoperative pain intensity. Pain scores included mean and standard deviation (SD) at the time of measurement. We included postoperative opioid consumption data only from those studies that performed IV or oral administration of opioids, but no restriction was applied to the mode of administration. We also excluded studies that reported the time to the first request for opioid analgesics or the number of times opioid analgesics was required. When studies used opioid analgesics other than morphine, the doses were converted into morphine equivalents, and the cumulative consumption at the time of measurement was recorded.

\section{Statistical analysis}

\section{Summary measures}

Opioid consumption data were extracted as mean and SD of the cumulative morphine consumption in the early and late periods. To measure pain intensity, we extracted the mean and SD of the pain scores of the early and late periods.

\section{Synthesis of results}

Changes in continuous outcomes, such as postoperative pain intensity or dose of opioid consumption, were pooled as standardized mean differences (SMDs), because different scaling of outcome measurements were expected across trials. We also calculated the $95 \%$ CIs for all estimates. A random-effect model was used to pool the study results, taking into account possible variations in effect sizes across trials. The heterogeneity statistic Cochrane $\mathrm{Q}$ and its corresponding (df) and P value as well as Higgins' $I^{2}$ as a measure of heterogeneity were calculated. $\mathrm{P}<0.05$ was considered representative of statistically significant heterogeneity, and $I^{2}>50 \%$ was considered to represent significant heterogeneity.

Post-hoc subgroup analyses were performed where possible for each outcome to explore heterogeneity based on the different route of administration between clonidine (per oral) and dexmedetomidine (intravenous). Chisquared tests for heterogeneity were performed to identify the differences between subgroups.

Publication bias was not evaluated because only few studies less than 10 were included in this meta-analysis.

We performed a sensitivity analysis to evaluate the stability of the results. Each study included in the meta-analy- 
sis was deleted each time to reflect the influence of an individual data set on the pooled effect estimate. We performed all analyses using R 3.51 (R Foundation for Statistical Computing, Austria) and Review Manager (RevMan, version 5.3, The Cochrane Collaboration).

\section{RESULTS}

\section{Study selection and characteristics}

We retrieved 2,477 articles after the initial database search: PubMed/MEDLINE ( $\mathrm{n}=960)$, EMBASE ( $\mathrm{n}=651$ ), CENTRAL ( $\mathrm{n}=704)$, and Korean databases $(\mathrm{n}=162)$.

After removing 1,845 duplicate articles, primary selection was performed on 632 articles. First, we excluded 550 unrelated articles by analyzing their titles and abstracts. Thereafter, full-text reviews were conducted on 82 articles. Of these 82 full-text articles, 71 were excluded for the following reasons: general anesthesia not performed or combined with regional anesthesia $(n=7)$, cardiac surgery ( $n$ $=1$ ), neurosurgery wherein neurological symptoms could occur $(\mathrm{n}=1)$, continuous infusion during or after surgery or unclear injection time $(\mathrm{n}=18)$, administration via routes other than the IV or oral route $(n=7)$, used in combination with other drugs (i.e., ketamine, gabapentin, or remifentanil) $(n=3)$, did not report appropriate outcomes such as pain score or opioid consumption $(n=13)$, no placebo group or study protocol without results $(n=9)$, articles not in Korean or English ( $\mathrm{n}=4)$, duplicated study $(\mathrm{n}=3)$, and full text unavailable $(\mathrm{n}=5)$. Finally, ROB evaluation and data extraction were performed on 11 studies [5-7,10-17]. Three studies [7,11,14] that did not report SD values were excluded from the meta-analysis of pain intensity (Fig. 1).

In all studies, alpha-2 agonists were administered via the oral $[6,13,16]$ or IV route $[5,7,10-12,14,15,17]$, but the doses and timing of injection differed among the studies (in most studies, the timing was between $2 \mathrm{~h}$ and right before anesthesia induction; in study of Pawlik et al. [13], the timing was the evening before and $2 \mathrm{~h}$ before anesthesia induction). Moreover, the type of opioids used differed across studies (morphine [14,17], fentanyl [7,11,15], and pethidine [6]). The frequency and interval of measurement of the pain score also varied across studies (ranging from every 30 min to once daily) (Table 1 ).

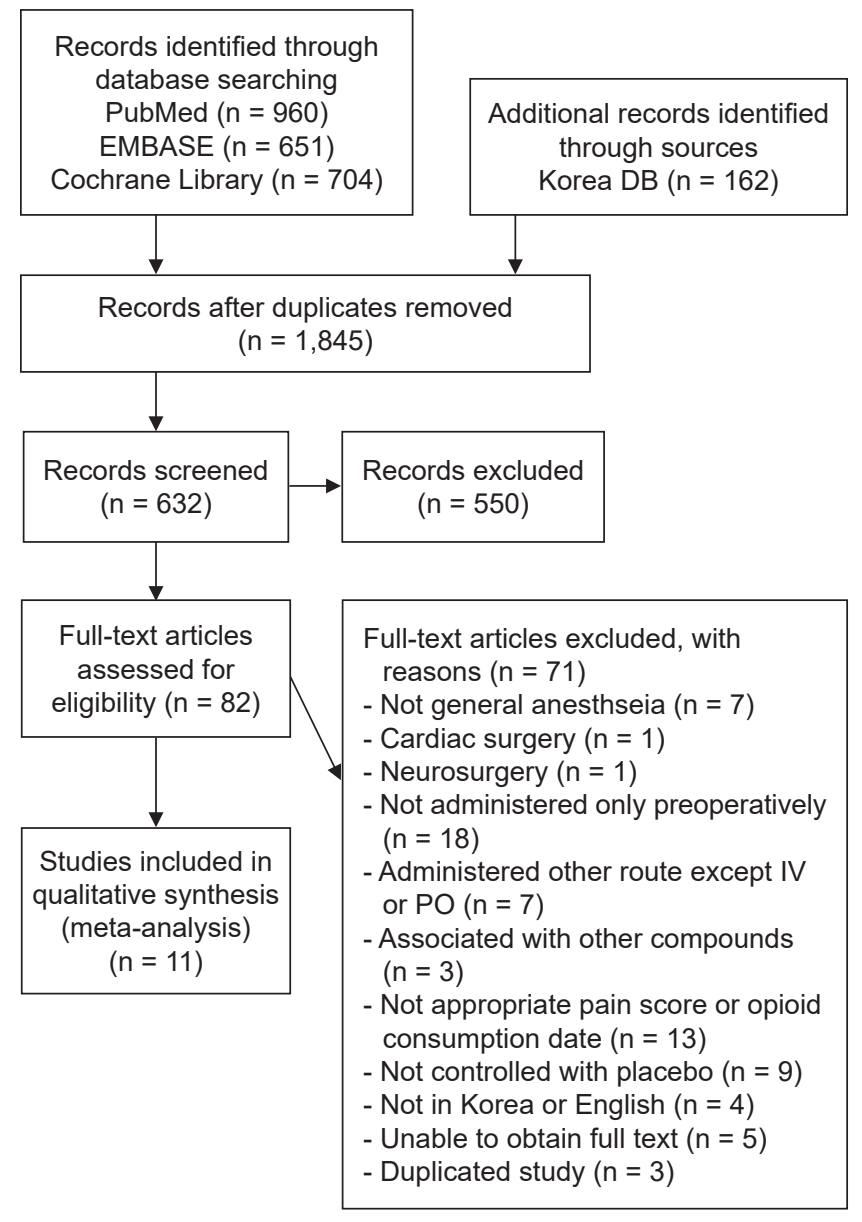

Fig. 1. Flow diagram of search strategy and study selection. DB: database, IV: intravenous, PO: postoperative.

\section{Quality assessment of the included studies (risk of bias within studies)}

The ROB evaluation revealed an overall low risk of bias (Figs. 2, 3).The proportion of studies assessed as "unclear" was $18.2 \%$ in random sequence generation, $27.3 \%$ in allocation concealment, $27.3 \%$ in blinding of participants and personnel, $9.1 \%$ in incomplete outcome data, and $18.2 \%$ in other bias. Random sequence generation was unclear in two studies $[6,10]$ that did not specify a randomization method. Allocation concealment was unclear in three studies $[6,10,11]$ that did not mention the specific allocation concealment method. Three studies that did not describe the blinding method for placebos were also rated as "unclear" $[6,13,16]$. The risk of incomplete outcome data was unclear in the study by Lee et al. [7], because the dropout rate was 5 to $20 \%$. Among the risk assessments of other biases mentioned in the Materials and Methods section, a 
power analysis to calculate the required sample size was not performed by Mizrak et al. [14], leading to a high risk of bias. The proportion of studies assessed as "unclear" was $54.5 \%$ in blinding of outcome assessment and $90.9 \%$ in selective reporting. Six studies inaccurately described whether blinding of outcome assessment was implemented $[6,7,10,11,15,16]$. Most studies did not provide information about Web-based study registration sites, except for one study [16].

\section{Effects on postoperative pain and opioid consump- tion}

The early period opioid consumption data extracted from two studies $[10,11]$ were data collected from the post-anesthesia care unit (PACU) instead of those collected up to $6 \mathrm{~h}$. The early period pain score data from two other studies $[12,13]$ were data collected from the PACU instead of those collected up to $6 \mathrm{~h}$ after surgery. Moreover, another study [6] analyzed pain score data measured at the 2nd hour. For the late period pain score analysis, data collected up to $8 \mathrm{~h}$ after surgery were extracted from one study [13] instead of those collected up to $24 \mathrm{~h}$ after surgery.

\section{Opioid consumption for early postoperative pain $(6 \mathrm{~h}$ after surgery)}

A meta-analysis synthesizing data from five effect sizes from five studies $[7,10,11,15,17]$ ( $n=236 ; 118$ in the experimental group and 118 in the control group) suggested that opioid consumption for early postoperative pain was significantly reduced in the preoperative alpha-2 agonist group (SMD, $-0.52 ; 95 \%$ CI [-0.90 to -0.14$]$; $\mathrm{P}=0.093 ; I^{2}=$ $50 \%$; Fig. $4 \mathrm{~A})$.

The test for subgroup differences indicated that there is no statistically significant subgroup effect $(\mathrm{P}=0.63$, analysis not presented), suggesting that the route of administration does not modify the effect of alpha-2 agonist in comparison to control drug on the opioid consumption for early postoperative pain.

\section{Opioid consumption for late postoperative pain $(24 \mathrm{~h}$ after surgery)}

We performed a meta-analysis synthesizing data from three studies $[6,7,17](\mathrm{n}=202 ; 89$ in the experimental group and 113 in the control group), which suggested that opioid consumption for late postoperative pain was significantly reduced in the preoperative alpha- 2 agonist group

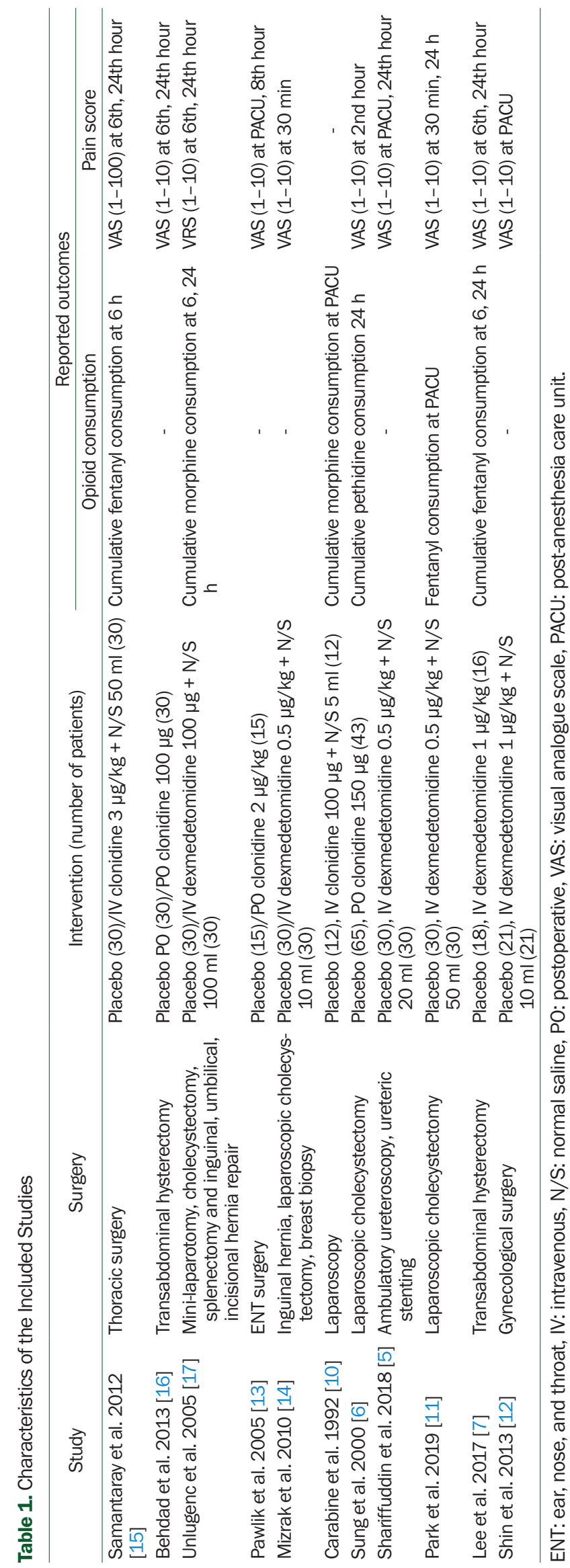




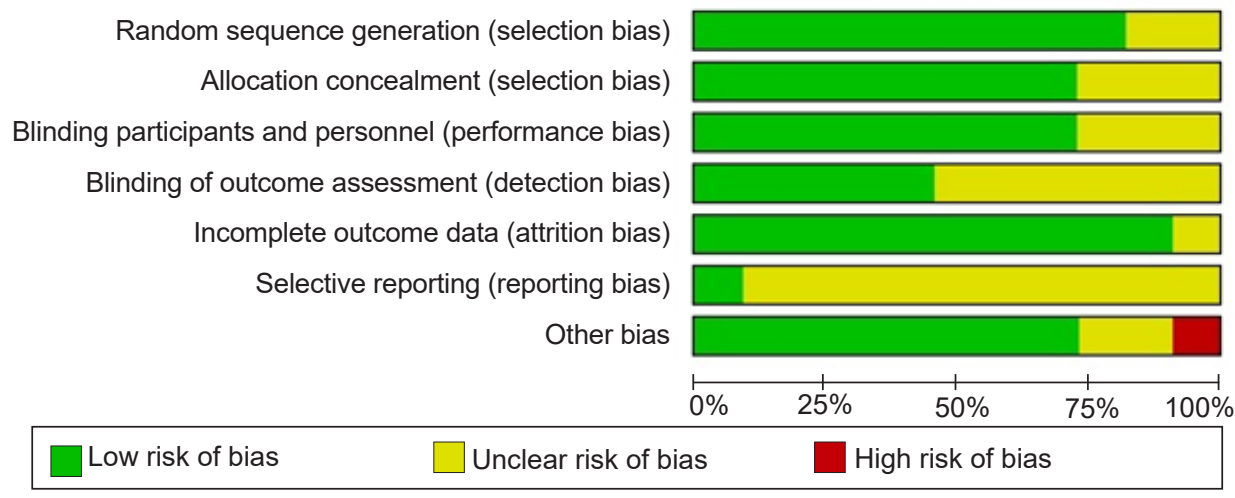

Fig. 2. Risk of bias graph: review authors' judgements about each risk of bias item presented as percentages across all included studies.

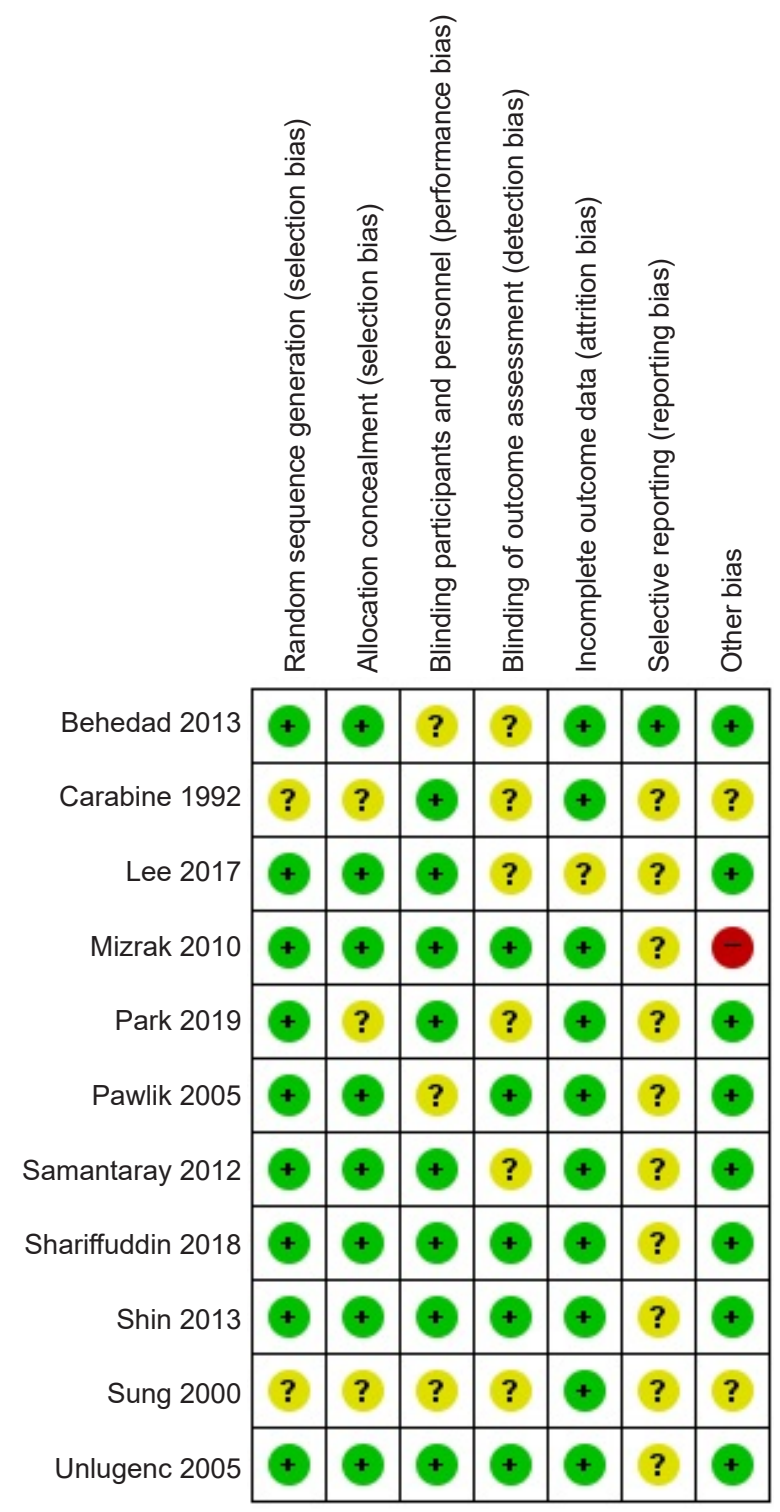

Fig. 3. Risk of bias summary: review authors' judgements about each risk of bias item for each included study.
(SMD, $-0.68 ; 95 \%$ CI $[-1.27$ to -0.09$] ; \mathrm{P}=0.025 ; I^{2}=73 \%$; Fig. 4B).

A subgroup analysis comparing the effect in postoperative opioid consumption in late period ( $24 \mathrm{~h}$ ) was not possible due to there being too few trials in this category.

\section{Pain score for early postoperative pain (6 $\mathrm{h}$ after surgery)}

A meta-analysis synthesizing data from seven studies $[5,6,12,13,15-17]$ ( $n=418 ; 199$ in the experimental group and 219 in the control group) suggested that the pain score for early postoperative pain was significantly reduced in the preoperative alpha-2 agonist group (SMD, $-0.50 ; 95 \%$ CI [-0.78 to -0.21$]$; P = 0.058; $I^{2}=51 \%$; Fig. $4 \mathrm{C}$ ).

The test for subgroup differences indicated that there is no statistically significant subgroup effect $(P=0.86$, analysis not presented), suggesting that the route of administration does not modify the effect of alpha- 2 agonist in comparison to control drug on the early postoperative pain.

\section{Pain score for late postoperative pain ( $24 \mathrm{~h}$ after surgery)}

Five studies $[5,13,15-17]$ ( $\mathrm{n}=268$; 135 in the experimental group and 133 in the control group) reported data on the pain score for late postoperative pain. Preoperative alpha-2 agonists significantly reduced the postoperative pain score (SMD, - 0.44; 95\% CI [-0.86 to -0.03 ]; P $=0.026 ; I^{2}=64 \%$; Fig. 4D).

The test for subgroup differences indicated that there is no statistically significant subgroup effect $(P=0.53$, analysis not presented), suggesting that the route of administration does not modify the effect of alpha-2 agonist in comparison to control drug on the late postoperative pain. 


\begin{tabular}{|c|c|c|c|c|c|c|c|}
\hline \multirow[b]{2}{*}{ Study or Subgroup } & \multicolumn{3}{|c|}{ alpha2-agonist } & \multicolumn{3}{|c|}{ Control } & \multirow[b]{2}{*}{ Weight } \\
\hline & Mean & SD & Total & Mean & SD & Total & \\
\hline Carabine 1992 & 5.8 & 4 & 12 & 8.3 & 4 & 12 & $13.9 \%$ \\
\hline Lee 2017 & 23.41 & 4.57 & 16 & 26.24 & 5.25 & 18 & $17.4 \%$ \\
\hline Park 2019 & 6.58 & 1.07 & 30 & 6.68 & 1.09 & 30 & $23.6 \%$ \\
\hline Samantaray 2012 & 12 & 6.9 & 30 & 15 & 11.8 & 28 & $23.1 \%$ \\
\hline Unlugenc 2005 & 17.6 & 7.83 & 30 & 25.6 & 6.25 & 30 & $22.0 \%$ \\
\hline Total $(95 \% \mathrm{Cl})$ & & & 118 & & & 118 & $100.0 \%$ \\
\hline $\begin{array}{l}\text { Heterogeneity: Tauz } \\
\text { Test for overall effec }\end{array}$ & $\begin{array}{l}0.09 ; \mathrm{Cr} \\
Z=2.69\end{array}$ & $\begin{array}{l}i^{2}=7 \\
(P=0\end{array}$ & $\begin{array}{l}\text { 7, df= } \\
007)\end{array}$ & $4(\mathrm{P}=$ & $0.093) ;$ & $F^{2}=50^{\circ}$ & \\
\hline
\end{tabular}

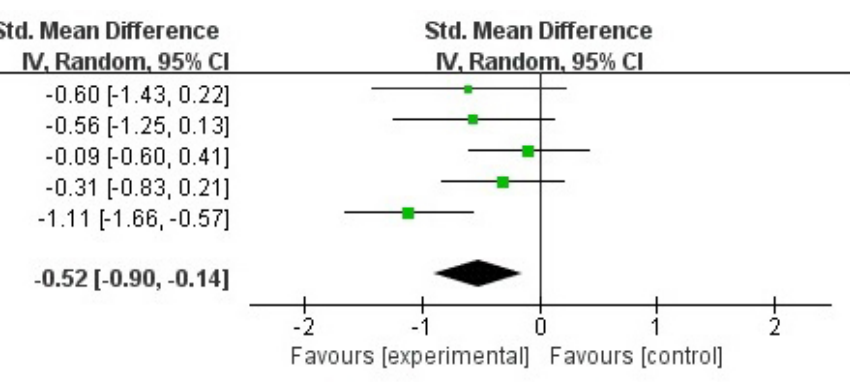

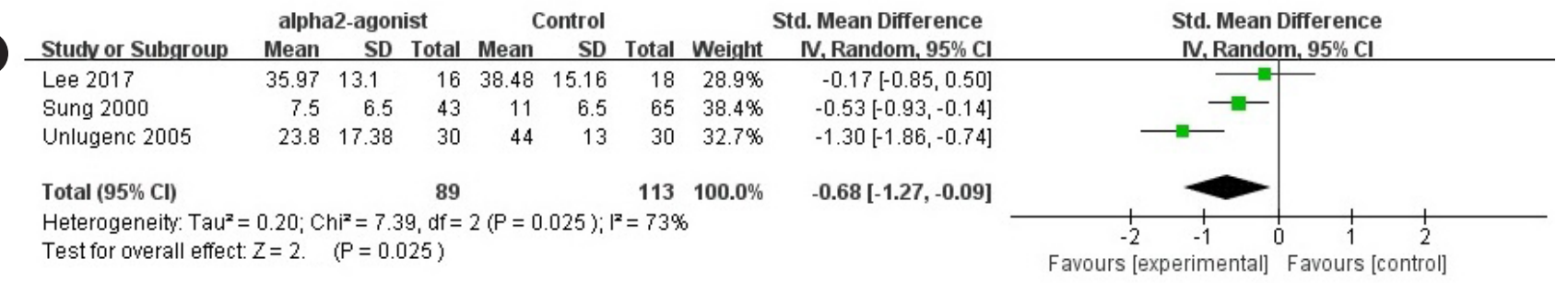

\begin{tabular}{lrrrrrrr} 
& \multicolumn{3}{c}{ alpha2-agonist } & \multicolumn{3}{c}{ Control } & \\
Study or Subgroup & Mean & SD & Total & Mean & SD & Total & Weight \\
\hline Behdad 2013 & 6.2 & 0.71 & 30 & 6.6 & 1.1 & 30 & $15.0 \%$ \\
Pawlik 2005 & 2.1 & 1.4 & 15 & 2.8 & 1.8 & 15 & $10.1 \%$ \\
Samantaray 2012 & 2.5 & 4.3 & 30 & 2.8 & 1.3 & 28 & $14.9 \%$ \\
Shariffuddin 2018 & 1 & 1.84 & 30 & 2.63 & 2.78 & 30 & $14.7 \%$ \\
Shin 2013 & 5 & 1.8 & 21 & 6.5 & 1 & 21 & $11.7 \%$ \\
Sung 2000 & 2.4 & 1.1 & 43 & 3.3 & 1 & 65 & $18.3 \%$ \\
Unlugenc 2005 & 1 & 1 & 30 & 1 & 1 & 30 & $15.2 \%$
\end{tabular}

Std. Mean Difference

Std. Mean Difference

V. Random, $95 \% \mathrm{Cl}$

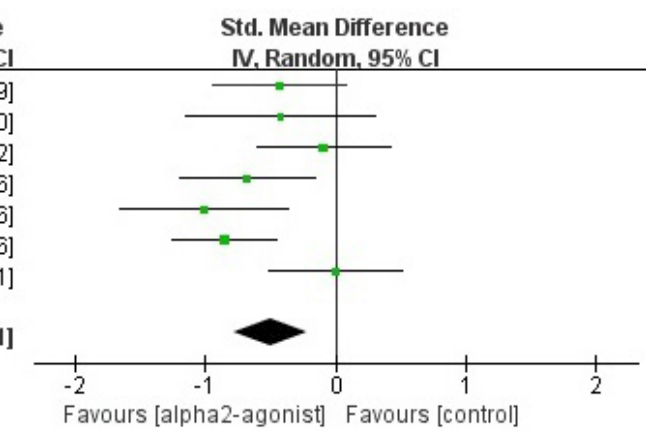

Total $(95 \% \mathrm{Cl}) \quad 199 \quad 219 \quad 100.0 \%$

Heterogeneity: $\mathrm{Tau}^{2}=0.07 ; \mathrm{Chi}^{2}=12.20, \mathrm{df}=6(\mathrm{P}=0.058) ; \mathrm{I}^{2}=51 \%$

Test for overall effect: $Z=3.39(P=0.0007)$

$-0.43[-0.94,0.09]$

$-0.42[-1.15,0.30]$

$-0.09[-0.61,0.42]$

$-0.68[-1.20,-0.16]$

$-1.01[-1.66,-0.36]$

$-0.86[-1.26,-0.46]$

$0.00[-0.51,0.51]$

$-0.50[-0.78,-0.21]$

Favours [alpha2-agonist] Favours [control]

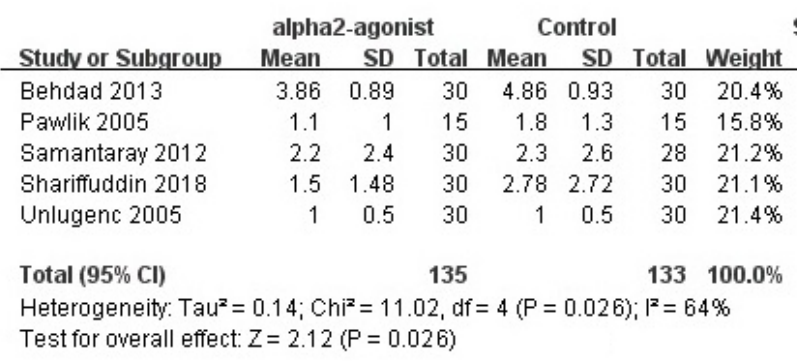

Std. Mean Difference

IV, Random, $95 \% \mathrm{Cl}$ Std. Mean Difference

$-1.08[-1.63,-0.54]$

$-0.59[-1.32,0.15]$

$-0.04[-0.55,0.48]$

$-0.58[-1.09,-0.06]$

$0.00[-0.51,0.51]$

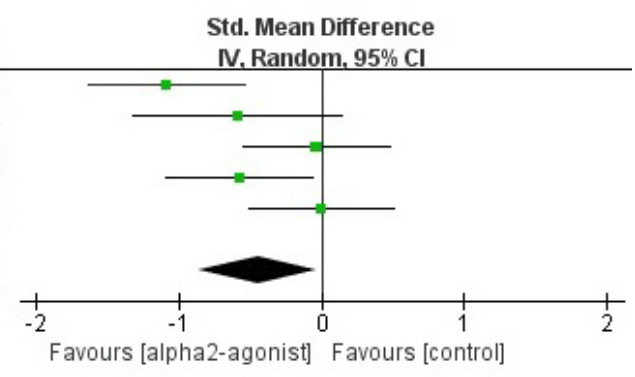

Fig. 4. Forest plot diagram showing the effect of alpha-2 agonist on postoperative pain intensity and opioid consumption. (A) Early period opioid consumption. (B) Late period opioid consumption. (C) Early period pain score. (D) Late period pain score. Std. Mean difference: standardized mean difference, IV: intravenous, Cl: confidence interval, SD: standard deviation.

\section{Sensitivity analysis}

Opioid consumption in the early postoperative period was $38 \%$ lower (SMD, -0.32 ; 95\% CI [-0.62 to -0.02 ]; $\mathrm{P}=$ 0.036) than the pooled estimate effect size (SMD, -0.52; 95\% CI [-0.90 to -0.14$] ; \mathrm{P}=0.007$ ) after omitting one trial [17]. Opioid consumption in the late postoperative period was $35 \%$ lower (SMD, $-0.44 ; 95 \%$ CI [ -0.78 to -0.10$]$; $\mathrm{P}=$ 0.010 ) than the pooled estimate effect size (SMD, -0.68; $95 \%$ CI [ -1.27 to -0.09$] ; \mathrm{P}=0.025$ ) after omitting one trial [17]. The pain scores in the early and late postoperative pe- riods were not statistically significantly different between the alpha-2 agonist group and control group after omitting one trial each $[16,17]$, which had a great influence on heterogeneity (results not shown).

\section{DISCUSSION}

In this meta-analysis, we evaluated the effect of preoperative administration of alpha- 2 agonists on total opioid consumption for postoperative analgesia and postoperative pain score. We found that opioid consumption and 
pain score were significantly lower in both the early and late periods. This may be due to a preemptive analgesic effect, anxiolytic effect, analgesic-sparing effect, or a residual additive effect of alpha-2 agonists $[5,17]$.

Recently, intraoperative administration of alpha-2 agonists has mainly been used for multimodal analgesia [1] According to the results of a meta-analysis conducted by Schnabel et al. [18], intraoperative use of dexmedetomidine leads to lower postoperative pain intensity and reduced opioid consumption. Therefore, in our analysis, why did the analgesic effect continue after surgery when alpha- 2 agonists were administered before surgery? This was the reason why we aimed to investigate whether preoperative administration of alpha- 2 agonists affects postoperative pain as does intraoperative administration of alpha- 2 agonists. Intraoperative or postoperative administration of alpha-2 agonists is likely more beneficial when considering only the duration of drug administration for postoperative pain management. However, this was difficult to analyze because each included study used different types of anesthetics, analgesics, and surgeries, as well as different durations of surgery. First, this could be considered a result of reducing preoperative anxiety in patients. Preoperative alpha-2 agonists are frequently used to reduce a patient's anxiety, which is also associated with a patient's preoperative well-being and decreased levels of postoperative pain [19]. Moreover, the preemptive effects could already have occurred as a result of the administration of alpha-2 agonists before surgical stimulation $[19,20]$.

This meta-analysis has several limitations. The number of studies included was quite small. To maintain good quality, only complete RCTs and studies published in English and Korean were included in this meta-analysis, thereby resulting in the small number of studies. Moreover, most studies had small sample sizes and some studies did not report postoperative pain and opioid consumption as primary endpoints. Since the types of anesthetics and analgesics used in each study were different, they could possibly have affected the endpoints we were measuring. In addition, the weight of the results of the meta-analysis was not high because of the inclusion of few studies that were conducted in accordance with our inclusion criteria.

Although not directly analyzed in this study, because opioid usage and opioid-related side effects increase proportionally [21], preoperative administration of alpha-2 agonists can be expected to reduce opioid-related side effects, as suggested by the results of this meta-analysis [22-24].
Furthermore, we did not distinguish between dexmedetomidine and clonidine when analyzing alpha-2 agonists. Because these two drugs have different selectivity for alpha-2 adrenal receptors, their effects may be different.

In summary, we demonstrated that preoperative IV or oral administration of alpha-2 agonists, such as clonidine and dexmedetomidine, may reduce postoperative opioid consumption and pain intensity until $24 \mathrm{~h}$. However, high degree of heterogeneity due to variations in the study method and small sized studies, limits the recommendations in the preoperative administration of alpha-2 agonists. Future powered large RCTs are required to increase the certainty of evidence on the effect in reducing postoperative opioid consumption and pain intensity.

\section{CONFLICTS OF INTEREST}

No potential conflict of interest relevant to this article was reported.

\section{AUTHOR CONTRIBUTIONS}

Conceptualization: Sangseok Lee. Study design: Ji Youn Ju, Kye-Min Kim, Sangseok Lee. Data acquision: Ji Youn Ju, Kye-Min Kim. Data analysis: Ji Youn Ju, Sangseok Lee. Writing_original draft: Ji Youn Ju, Kye-Min Kim, Sangseok Lee. Writing-review \& editing: Ji Youn Ju, Kye-Min Kim, Sangseok Lee.

\section{ORCID}

Ji Youn Ju, https://orcid.org/0000-0001-9069-4189

Kye-Min Kim, https://orcid.org/0000-0003-1298-7642

Sangseok Lee, https://orcid.org/0000-0001-7023-3668

\section{REFERENCES}

1. Rasmussen ML, Mathiesen O, Dierking G, Christensen BV, Hilsted KL, Larsen TK, et al. Multimodal analgesia with gabapentin, ketamine and dexamethasone in combination with paracetamol and ketorolac after hip arthroplasty: a preliminary study. Eur J Anaesthesiol 2010; 27: 324-30.

2. Khan ZP, Ferguson CN, Jones RM. Alpha-2 and imidazoline receptor agonists. Their pharmacology and therapeutic role. Anaesthesia 1999; 54: 146-65.

3.Kamibayashi T, Maze M. Clinical uses of alpha2-adrenergic agonists. Anesthesiology 2000; 93: 1345-9. 
4. Blaudszun G, Lysakowski C, Elia N, Tramèr MR. Effect of perioperative systemic $\alpha 2$ agonists on postoperative morphine consumption and pain intensity: systematic review and meta-analysis of randomized controlled trials. Anesthesiology 2012; 116: 1312-22.

5. Shariffuddin II, Teoh WH, Wahab S, Wang CY. Effect of single-dose dexmedetomidine on postoperative recovery after ambulatory ureteroscopy and ureteric stenting: a double blind randomized controlled study. BMC Anesthesiol 2018; 18: 3.

6. Sung CS, Lin SH, Chan KH, Chang WK, Chow LH, Lee TY. Effect of oral clonidine premedication on perioperative hemodynamic response and postoperative analgesic requirement for patients undergoing laparoscopic cholecystectomy. Acta Anaesthesiol Sin 2000; 38: 23-9.

7. Lee KY, Lee WY, Kim KM, Yoo BH, Lee S, Lim YH, et al. The effect of pre-anesthetic administration of dexmedetomidine on the consumption of opioids in postoperative gynecologic patients. Anesth Pain Med 2017; 12: 37-41.

8. Moher D, Liberati A, Tetzlaff J, Altman DG; PRISMA Group. Preferred reporting items for systematic reviews and meta-analyses: the PRISMA statement. BMJ 2009; 339: b2535.

9. Viera AJ, Garrett JM. Understanding interobserver agreement: the kappa statistic. Fam Med 2005; 37: 360-3.

10. Carabine UA, Allen RW, Moore J. Partial attenuation of the pressor response to endotracheal intubation. A comparison of the effects of intravenous clonidine and fentanyl. Eur J Anaesthesiol 1992; 9: 325-9.

11. Park H, Kim HS, Kim JW, Lee GG, Park DH, Jeong CY, et al. Effects of preanesthetic single administration of dexmedetomidine on the remifentanil and propofol requirement during laparoscopic cholecystectomy. Anesth Pain Med 2019; 14: 2934.

12. Shin HW, Yoo HN, Kim DH, Lee H, Shin HJ, Lee HW. Preanesthetic dexmedetomidine $1 \mu \mathrm{g} / \mathrm{kg}$ single infusion is a simple, easy, and economic adjuvant for general anesthesia. Korean J Anesthesiol 2013; 65: 114-20.

13. Pawlik MT, Hansen E, Waldhauser D, Selig C, Kuehnel TS. Clonidine premedication in patients with sleep apnea syndrome: a randomized, double-blind, placebo-controlled study. Anesth Analg 2005; 101: 1374-80.

14. Mizrak A, Koruk S, Bilgi M, Kocamer B, Erkutlu I, Ganidagli S, et al. Pretreatment with dexmedetomidine or thiopental decreas- es myoclonus after etomidate: a randomized, double-blind controlled trial. J Surg Res 2010; 159: e11-6.

15. Samantaray A, Rao MH, Chandra A. The effect on post-operative pain of intravenous clonidine given before induction of anaesthesia. Indian J Anaesth 2012; 56: 359-64.

16. Behdad S, Ayatollahi V, Yazdi AG, Mortazavizadeh A, Niknam F. Effect of oral low dose clonidine premedication on postoperative pain in patients undergoing abdominal hysterectomy: a randomized placebo controlled clinical trial. Rev Med Chir Soc Med Nat Iasi 2013; 117: 934-41.

17. Unlugenc H, Gunduz M, Guler T, Yagmur O, Isik G. The effect of pre-anaesthetic administration of intravenous dexmedetomidine on postoperative pain in patients receiving patient-controlled morphine. Eur J Anaesthesiol 2005; 22: 38691.

18. Schnabel A, Meyer-Frießem CH, Reichl SU, Zahn PK, PogatzkiZahn EM. Is intraoperative dexmedetomidine a new option for postoperative pain treatment? A meta-analysis of randomized controlled trials. Pain 2013; 154: 1140-9.

19. Kalkman CJ, Visser K, Moen J, Bonsel GJ, Grobbee DE, Moons KG. Preoperative prediction of severe postoperative pain. Pain 2003; 105: 415-23.

20. Kelly DJ, Ahmad M, Brull SJ. Preemptive analgesia I: physiological pathways and pharmacological modalities. Can J Anaesth 2001; 48: 1000-10.

21. Roberts GW, Bekker TB, Carlsen HH, Moffatt CH, Slattery PJ, McClure AF. Postoperative nausea and vomiting are strongly influenced by postoperative opioid use in a dose-related manner. Anesth Analg 2005; 101: 1343-8.

22. Liu SS, Strodtbeck WM, Richman JM, Wu CL. A comparison of regional versus general anesthesia for ambulatory anesthesia: a meta-analysis of randomized controlled trials. Anesth Analg 2005; 101: 1634-42.

23. Marret E, Kurdi O, Zufferey P, Bonnet F. Effects of nonsteroidal antiinflammatory drugs on patient-controlled analgesia morphine side effects: meta-analysis of randomized controlled trials. Anesthesiology 2005; 102: 1249-60.

24. Gurbet A, Basagan-Mogol E, Turker G, Ugun F, Kaya FN, Ozcan B. Intraoperative infusion of dexmedetomidine reduces perioperative analgesic requirements. Can J Anaesth 2006; 53: 646-52. 
Appendix 1. Example of electronic database searching strategy for MEDLINE

\#16. \#14 AND \#15

\#15. random*

\#14. \#13 AND [english]/lim

\#13. \#5 AND \#11 AND \#12

\#12. postop* OR postane* OR 'post op*' OR 'post ane*' OR postanae* OR 'post anae*'

\#11. \#6 OR \#7 OR \#8 OR \#9 OR \#10

\#10. pca OR 'patient controlled analgesia'

\#9. analgesic*

\#8. fentanyl

\#7. morphine

\#6. opioid*

\#5. \#1 OR \#2 OR \#3 OR \#4

\#4. (alpha AND 2 OR 'alpha-2' OR a2 OR 'a 2') AND agonist*

\#3. precedex

\#2. dexmedetomidine

\#1. clonidine 